\title{
Las ciudades invisibles: primeros núcleos españoles en América
}

\section{Alfredo Cordiviola \\ Professor adjunto e pesquisador do Departamento \\ de Letras e do Programa de Pós-Graduaçāo em \\ Letras da Universidade Federal de Pernambuco}

Resumen: Navidad e Isabela, los dos primeros núcleos urbanos creados por españoles en América, surgen entre 1492 y 1494 , pero se desvancecen y son definitivamente abandonados poco después. Fundados en la isla Española, esos efimeros poblados serían así el marco inicial de lo que vendría a ser el proyecto colonizador. La historia de Navidad y de Isabela nos deja entrever los conflictos y las propuestas que definen la etapa antillana de la conquista, y anuncia un programa de ocupación sistemática del territorio que sería firmemente cumplido en las décadas siguientes. A partir de la lectura de los textos colombinos, $y$ de otras fuentes relativas al segundo viaje de exploración de América, este ensayo describe las circunstancias que marcaron la fundación y la rápida decadencia de esos primeros asentamientos europeos en el Nuevo Mundo.

Palabras-llave: ciudad; época colonial; literatura hispano-americana
Resumo: Navidad e Isabela, os dois primeiros núcleos urbanos criados pelos espanhóis na América, surgem entre 1492 e 1494, mas se desvancecem e são definitivamente abandonados pouco depois. Fundados na ilha Española, esses efêmeros povoados seriam assim o marco inicial do que viria a ser o projeto colonizador. A história de Navidad e de Isabela permite entrever os conflitos e as propostas que definem a etapa antilhana da conquista, e anuncia já um programa de ocupação sistemática do território que seria firmemente cumprido nas décadas seguintes. A partir da leitura dos textos colombianos, e de outras fontes relativas à segunda viagem de exploraçāo da América, este ensaio descreve as circunstâncias que marcaram a fundaçāo e a rápida decadência desses primeiros assentamientos europeus no Novo Mundo.

Palavras-chave: cidade; época colonial; literatura hispano-americana 
1. La fundación de ciudades suele estar asociada a las expectativas que despierta el género épico. Allí donde no hay nada, o donde se ha destruido lo que antes había, se imagina aquello que estará por venir, se crean las bases para algo que sólo al futuro le corresponderá confirmar o negar. Si el momento mismo de la fundación alude a fechas y nombres propios precisos (que después serán repetidamente celebrados para reafirmar a cada año y en cada monumento aquella visión original), en realidad toda fundación no pasa de ser uma apuesta lanzada contra un futuro que es siempre incierto e inasible. En el instante de su fundación, toda ciudad, en su precariedad e indefensión, remite a una otra ciudad, por el momento ausente y fantasmática, porque todavía no existe. Una ciudad hipotética, que podría declinar o desaparecer, que podrá o no desarrollarse, según vaya contando con el amparo de diversos factores de orden político, económico e histórico.

Para que ello ocurra, primero es necesario (según enfatizan las mitologias de fundación) que haya un gesto que revele las habilidades de un estratega, aquel que sepa definir el lugar y el momento exactos en que la fundación deba ser realizada. El puerto más seguro, la latitud más propicia, la región más transparente son así adivinadas por la perspicacia del soldado astuto o del marino avizor. Pero también no es infrecuente que los dioses quieran participar activamente en esa astucia, como ayudantes o idealizadores. Apariciones, mandatos, instrucciones divinas sitúan la instancia fundadora en otra dimensión. Así, relatos tan disímiles como los que narran por ejemplo la fundación de Tebas y de Cuzco remiten a una firme voluntad divina que guia los pasos de sus emisarios, para que cumplan un destino previamente trazado. La vaca que Cadmus encuentra cerca del oráculo de Delfos tendrá en algum momento que descansar, y allí deberá ser fundada la ciudad, de la misma forma que los enviados de Viracocha deben ir hundiendo en su caminata civilizatoria rumbo al norte una vara de oro, hasta que desparezca por completo en la tierra para indicar la localización del ombligo del mundo. En ambos casos, la fundación ha sido determina- 
da por los dioses, que ya reservan para la futura ciudad un esplendor que la torne digna de sus ambiciones y que justifique su propia función en el universo.

Ciudades son inventadas por múltiples motivos: como marco civilizatorio contra las amenazas del entorno, por voluntad de organización contra el caos y la anarquía, como proyecto de establecer comunidades contra el desarraigo y el extravío, por decisión política y militar para adueñarse antes de zonas estratégicas o generosas en riquezas y codiciadas también por otras naciones. Fundar ciudades supone un gesto definitivo, cuyas consecuencias son al mismo tiempo inmediatas y evidentes (un nombre, una marca en la geografía, un punto en el mapa) y al mismo tiempo imprevisibles. En la América que los españoles van ocupando a partir de los ultimos años del siglo XV, sobran ejemplos de ese gesto fundacional que da nombres y delimita espacios a ser habitados. Como es sabido, en el breve período que va del final del siglo XV hasta mediados del XVI, prácticamente todas las principales ciudades hispanoamericanas serán fundadas. Santo Domingo, Santiago de Cuba, San Juan, Habana en la época antillana (1493-1519), Panamá, México, Puebla, Cartagena, Cuzco, Lima, Quito, Potosí, Bogotá, entre muchas otras, durante el reinado de Carlos V (hasta 1556) van consolidando un ciclo que será completado durante la segunda mitad del siglo, en el reinado de Felipe II (hasta 1598).

Fundar ciudades y poblarlas es la herramienta clave de la política española para perpetuar la conquista y establecer la firme y progresiva expansión del imperio. Las fundaciones obedecen a una serie de convenciones y procedimientos que pretenden dar un marco legal a la ocupación. Actas de fundación son autorizadas por escribanos y testigos, símbolos como cruces, rollos y marcos consagran la presencia física de los poderes temporales y espirituales, misas son celebradas para invocar la protección de los santos patronos. Con esas ceremonias, se define el trazado urbano que habrá de organizar el espacio ocupado. A medida que pasan los años, son redactadas en la corte una serie de instrucciones que 
estipulan los modos en que deben ser delineadas las plantas, con su plaza central y sus calles principales. Algunas de las más conocidas son la Instrucción dada a Pedrarias Dávila (1513), o la Ordenanza de Carlos v (1523), que establece claramente que

cuando hagan la planta del lugar, repártanlo por sus plazas, calles y solares a cordel y regla, comenzando desde la plaza mayor, y sacando desde ella calles a las puertas y caminos principales, y dexando tanto compás abierto que aunque la población vaya en gran crecimiento, se pueda siempre proseguir y dilatar en la misma forma. ${ }^{1}$

Planeadas racionalmente "a cordel y regla", las ciudades hispanoamericanas surgen a partir de la aplicación de principios de abstracción y sistematización, que aspiran a imprimir un orden sobre una geografía a ser dominada y explotada. Las ciudades representan el corazón mismo de ese orden, al exhibir una clara jerarquía social, evidenciada por los trazados reticulares y por sus centros simbólicos, representados por la plaza, donde habrán de ser puestas en escena las ceremonias oficiales, como las procesiones, los autos de fe o la aclamación de los virreyes.

Núcleos de ocupación, de control y de afirmación de poder, las ciudades en pocas décadas se transforman en piezas centrales para la consolidación del circuito comercial atlántico que surge en el siglo XVI, y que propicia una nueva internacionalización, sustentada por las redes que unen las costas americanas, africanas y europeas. Con el nacimiento de aquello que Mignolo denomina "sistema colonial moderno", surge también una nueva conciencia de universalidad basada en la idea de orbis cristiano, que se refleja y se conoce a sí misma en los mapas que por primera vez son capaces de ofrecer una visión unificada y global del planeta.

Como escenario donde se ensayan nuevas formas de cohesión social y se fragmentan o se anulan las relaciones vigentes en el mundo prehispánico, la esfera urbana, con sus ruínas y edificaciones, rapidamente se va trans-
${ }^{1}$ En Sanz Camañes, Porfírio. Las ciudades en la América

Hispana. Siglos XV al XVIII. Madrid: Silex, 2004, p. 37. 
formando en el espacio central de las interacciones entre europeos e indígenas. Allí, los hábitos culturales nativos perseguidos o parcialmente erradicados insisten en persistir y se transforman, mientras que los elementos cristianos se adaptan a nuevas realidades y adquieren sentidos diversos. En las ciudades geométricas y precarias se yuxtaponen la idea de castrum romano, las concepciones ideales del renacimiento y las organizaciones espaciales indígenas; los "pueblos de indios" en la periferia y los centros del poder español representados, alrededor de la plaza principal, por los palacios reales, las catedrales, los cabildos y las casas de los conquistadores.

Pero si en pocos años algunas de esas poblaciones se transforman en grandes urbes y adquieren status de capital, como Lima, por ejemplo, o aquellas que habían sido edificadas sobre las ruinas de ciudades prehispanicas, como Mexico o Cuzco, hay tambien algunos casos en que los poblados rápidamente son aniquilados por el fracaso y abandonados a su suerte. Uno de esos casos sería Buenos Aires, fundada por Pedro de Mendoza en 1536 y abandonada poco después debida a las insalvables penurias y la hambruna generalizada que les tocó padecer a los aventureros allí reunidos. Para ese entonces, el fracaso de la villa situada junto al rio de la Plata (que seria fundada por segunda vez en 1580) no había sido el único, y contaba con algunos antecedentes que remitían a los comienzos mismos de la presencia española en América. Cuatro décadas antes, ese había sido el mismo destino de las dos primeras poblaciones erigidas por europeos de este lado del Atlántico, el Fuerte de Navidad y la villa de la Isabela, ambas en la isla Española.

2. El dia de Navidad de 1492 fue particularmente ingrato para los navegantes que ya llevaban más de dos meses en tierras que después serían llamadas americanas. Por impericia de algún muchacho inexperiente, la nave capitana, Santa María, encalla definitivamente frente a las costas de la isla Española. Según la relación que Las Casas ofrece del primer viaje, 
Y a esto (dice él), vinieron tantas cosas a la mano, que verdaderamente, no fue aquél desastre, salvo gran ventura. Porque es cierto (dice él) que si yo no encallara, que yo fuera de largo sin surgir en este lugar, porque él está metido acá dentro en una grande bahía y en ella dos o tres restringas de bajas. Ni este viaje dejara aquí gente, ni aunque yo quisiera dejarla no les pudiera dar tan buen aviamento ni tantos pertrechos ni tantos mantenimientos ni aderezos para fortaleza. Y bien es verdad que mucha gente de esta que va aquí me habían rogado y hecho rogar que les quisiera dar licencia para quedarse. Agora tengo ordenado de hacer una torre y fortaleza, todo muy bien, y una grande cava, no porque crea que haya esto menester por esta gente, porque tengo dicho que con esta gente que yo traigo sojuzgaría toda esta isla, la cual creo que es mayor que Portugal, y más gente al doblo; mas son desnudos y sin armas y muy cobardes fuera de remedio. Mas es razón que se haga esta torre y se esté como se ha de estar, estando tan lejos de Vuestras Altezas, y porque conozcan el ingenio de la gente de Vuestras Altezas y lo que pueden hacer, porque con amor y temor le obedezcan; y así terminan tablas para hacer todas las fortalezas de ellas y mantenimientos de pan y vino para más de un año y simientes para sembrar y la barca de la nao y un calafete y un carpintero y un lombardero y un tonelero y muchos entre ellos hombres que desean mucho, por servicio de Vuestras Altezas y me hace placer, de saber de la mina adonde se coge el oro. Así que todo es venido mucho a pelo para que se faga este comienzo. ${ }^{2}$

Este párrafo es emblemático por varios motivos, pero principalmente porque alude a uma síntesis de deseos que se mantendrá presente en gran parte de los textos colombinos. El accidente, el infortunio (un navio que encalla y se pierde) son de alguna forma los instrumentos que tornam posible y potencializan esta confluencia de tres deseos. Confluencia que define este momento inicial para Colón, y que le permite encontrar un sentido último (y secreto) para su aventura. O mejor dicho, transforma una simple o compleja aventura marítima (pero aventura al fin) en un proyecto, con sus fines, cálculos y consecuencias.
${ }^{2}$ Diario del primer viaje de Colón, en Las Casas, Bartolomé. Obras completas, volumen 14.. Madrid: Alianza, 1989, p. 128-129. 
${ }^{3}$ Idem, ibidem, p. 126.

Si el episodio de la Santa Maria no ha sido un desastre, sino "una gran ventura" es porque por detrás del incidente se advierten los designios de la Providencia divina, una figura que irá cobrando cada vez mayor importancia en el imaginario colombino, como evidencian sus anotaciones que después serán recogidas en el llamado Libro de las profecías. La acción divina, que es irreprochable, aunque a veces pueda ser incomprensible, ha querido que el navío fuera destruido para que los primeros españoles pudiesen establecerse y encontrar resguardo en tierras desconocidas.

El deseo divino se complementa en el deseo manifiesto de los españoles que le "habían rogado para quedarse", para poder explorar las tierras interiores del Cibao (que podía ser Cipango), donde se decía que había oro. Y el triángulo se completa con el deseo de los indígenas, que puede ser inferido a partir de sus actitudes y disposiciones ("ellos aman a sus próximos como a si mismos, y tienen una habla la más dulce del mundo y mansa, y siempre con risa”, escribe Colón en su Diario. $\left.{ }^{3}\right)$. Para el Almirante, no cabe duda de que los indígenas abrazarían rápidamente el cristianismo, y que aceptarían "con amor y temor" la evangelización una vez que esta se organizara en forma sistemática. Esa presunción se basa en las alianzas puntuales, selladas por rituales y trueques, que Colón establece con el cacique Guacanagarí para asegurar la convivencia, y además en las consabidas idealizaciones que elabora a partir de la aparente inocencia y buena voluntad de los nativos.

Así, con los restos de la Santa María, y con la fuerza mancomunada de indígenas y españoles, se erige el fuerte Navidad. De esta manera se conforma la escena inicial de la ocupación, propiciada por el destino y enmarcada por un mutuo entendimiento entre las partes. "He tomado posesión”, escribe Colón en la Carta a Luís de Santángel, "de una villa grande, a la cual puse nombre la villa de Navidad; y en ella he hecho fuerza y fortaleza". Ya no se trataría aqui de una visita, ni de meros reconocimientos, ni de intercambios más o menos fugaces como los que se multiplican en los documentos que narran lo acontecido 
anterioremente durante el primer viaje. Ahora se trata de treinta y nueve hombres, en sus precarias moradías, que debían permanecer de este lado del Atlántico al menos hasta que volviese el almirante con sus navíos, y que tendrían por lo tanto que negociar y aprender a convivir con los otros y entre sí, en un lugar al que habían otorgado un nombre auspicioso y ejemplar: Navidad.

La posteridad supo imaginar ese momento em tonos casi idílicos. Tal vez una de las imágenes que mejor describe ese instante sea la estampa que aparece en la edición española (1851) de Vida y viajes de Cristóbal Colón de Washington Irving. ${ }^{4}$ El grabado, pautado por las convenciones pictóricas de la época, muestra, sobre el fondo de una naturaleza tropical y un cielo de grandes nubes, grupos numerosos de hombres febrilmente ocupados en la construcción de la fortaleza. Los contornos pocas veces permiten identificar si se trata de nativos o españoles, salvo en el primer plano, donde aparece claramente una figura con el brazo en alto y un sombrero como el que Colón luce en el retrato más o menos imaginario del renacentista Sebastiano del Piombo (sobre ese retrato, en verdad no se ha establecido fehacientemente que sea de Sebastiano del Piombo ni si realmente se refiere a Colón, pero ha perdurado en la memoria y fue reproducido muchas veces. Es probable que haya sido pintado en la década de 1520. Posteriormente, Theodore de Bry realizó un grabado a partir de esa imagen). Su brazo apunta al promontorio donde se levanta la torre principal, rodeada por murallas, y formada apenas por un conjunto de maderas que van ganando formas. Abajo, en las aguas, está anclada una carabela, con sus velas recogidas. Pocas visiones de la conquista podrían ser más optimistas que esta, que exhibe una voluntad sin fisuras y una cooperación plena entre antillanos y españoles en busca de un objetivo común.

Pero esa sociedad ilusoria, como se sabe, era más frágil que las esperanzas de consolidar acuerdos y complicidades. Casi um año despues de la creación del fuerte, cuando Colón retorna a América, encuentra el lugar devastado. Tras pasar por Dominica, Guadalupe, por las
${ }^{4}$ Ver Irving, Washington. Vida $y$ viajes de Cristóbal Colón. Madrid: Gaspar y Roig Editores, 1851, p. 49. 
${ }^{5}$ Ver Varela, Consuelo. Cristóbal Colón. Textos y documentos completos. Madrid: Alianza, 1997, p.243. El cacique Caonabó sería después capturado, y muere durante la navegación, cuando era llevado prisionero a España. islas que Colón denomina de las Once mil vírgenes y Puerto Rico, los navegantes llegan el 22 de noviembre de 1493 a La Española, donde divisan algunos cadáveres abandonados. En pocos días tendrían la confirmación de que todos los españoles habían muerto. Tres habían sido los principales factores que desencadenaron el fin de Navidad: la resistencia indígena, en este caso encabezada por el cacique Caonabo, que pretendía acabar con los abusos cometidos por los cristianos, la discordia interna y las rivalidades entre los propios españoles, y las enfermedades. En su "Carta Relación del segundo viaje", Colón registra el testimonio de un informante indígena enviado por el cacique Ocanaguarí sobre lo acaecido en Navidad:

Este me dixo [en] cómo la gente que yo avía dexado en la çiudad ovieron entre sí discordia y uno mató a otro y que Pedro, repostero de V. Al., se avía ido con una gran parte de jente para otro rey que se llama Cahonaboa, el cual posee tierra en que ay mucho oro; y un vizcaíno, que se llama Chacho, se avía ido con otros vizcaínos y moços; solamente avía quedado Diego de Arana de Córdoba con honze; y que unos tres se avían muerto de dolencia, qu'ellos mesmos deçían que era la causa el gran tracto de las mugeres, diziendo que, cuantos quedaron allí, que cada uno avía tomado cuatro mugeres, y no solamente estas les abastava, que les tomavan las muchachas. Y deçían qu'el comienço d'esta discordia fue que, luego que yo parti, cada uno no quiso entrar a obediencia ni apañar oro salvo para sí, sino Pedro, repostero, y Escobedo - a éste avía yo dexado el cargo de todas las cosas -. Y que los otros no entendían salvo con mugeres y moravan en casa d'ellas, y que Pedro y Escobedo mataron uno que se llamaba Jácome, y después se fueron con sus mugeres a este Cahonaboa; y dende a çierto tiempo bino este Cahonaboa y de noche puso fuego en la villa, la cual quemó toda que no quedó nada, de qu'es de aver piedad, porque otra poblaçión tan grande ni de $\tan$ hermosas casas e visto en todas las Yndias

El fuego y la discordia habían acabado con ese minúsculo villorio, que sólo en la hiperbólica imaginación colombina 
podía ser muy grande y contar con hermosas casas. Pero en el horizonte ya había otros barcos, y en breve la isla Española dejaría de ser para siempre eso que algunos españoles habían tenido la posibilidad de ver en los últimos meses de 1492.

3. La flota que había partido del puerto de Cádiz el miércoles 25 de septiembre de 1493 poco tenía em común con los tímidos navíos que Colón había comandado el año anterior en su primer viaje a América. Tres barcos apenas habían hecho la travesía em 1492; ahora serían diecisiete, que transportaban aproximadamente unos 1500 pasajeros. Como diría Pedro Martir en su carta a Ascanio Visconti, el Almirante había dispuesto “(...) toda clase de artífices para edificar una ciudad nueva. Lleva consigo animales y semillas de toda especie". ${ }^{6}$ El porte de la flota, y el carácter heterogéneo y representativo de la tripulación, ya indicaba que este era un proyecto oficial de la corte de los Reyes Católicos cuyo objetivo era ocupar y poblar el territorio. Además de los marineros y de los soldados, había en estos barcos gentes cuyos oficios serían vitales para el desarrollo de la vida cotidiana: carpinteros, boticarios, escribanos, sastres, campesinos, mineros, plateros, herreros, calafateros componen entre otros una lista que aspira a recrear en América el tejido social de las villas españolas. Como afirma León Guerrero, "El segundo viaje, al ser de asentamiento y población, supone un traslado de una representación de todos los estamentos de la sociedad, así como de todos aquellos oficios necesarios para el establecimiento de ciudades en la nuevas tierras". ${ }^{7}$ Mujeres, niños, sacerdotes (entre ellos el nuncio papal Bernardo Boyl y Fray Ramón Pané, autor más tarde de una conocida Relación), el cartógrafo Juan de la Cosa y escuderos del Almirante, como su hermano Diego, son algunos de los nombres destacados de un contingente que, en grande parte, tenía por misión establecerse y preparar el camino para la llegada de otros flujos inmigratorios hacia América.

A toda esa sociedad em miniatura, con sus jerarquías y sus estamentos, le correspondería ser prota-
${ }^{6}$ Pedro Martir de Anglería, Epístola 138, en Cartas sobre el Nuevo Mundo. Madrid: Polifemo, 1990, p. 34.

${ }^{7}$ La excelente tesis doctoral de Maria Monserrat León Guerrero, El segundo viaje colombino (Alicante: Biblioteca Virtual Miguel de Cervantes, 2002, p. 169 ), presenta um análisis detallado de la tripulación. Puede consultarse em www.cervantesvirtual 
gonista de una empresa cuyo marco inaugural en tierras americanas es la fundación de una villa, en la misma isla de la Española donde quedaban las ruinas del fuerte Navidad. Ya no se trataba, como este, de um mero puesto de vigilancia ni de una guarnición provisoria, sino de una ciudad que los propios reyes, en sus Instrucciones de mayo de 1493, habían encomendado erigir.

Tras el reconocimiento de la isla, cumplido por las expediciones de Diego Márquez, Gines de Corbalán y Alonso de Ojeda, y para no incurrir en mayores dilaciones, a fines de diciembre Colón elige para la fundación un sitio en la costa norte de la isla, junto al río Bajabonico. El paraje era vecino a "ciertas casas de indios", y próximo a la región de Cibao, donde, según los informantes nativos, había grandes cantidades de oro. Esta vez el nombre del emplazamiento no obedece a la fecha de su creación, como el de Navidad, sino que presta un explícito homenaje a la reina de Castilla: La Isabela.

Evocar nombres familiares, como se sabe, es un recurso habitual de los navegantes, y en los textos colombinos son numerosas las referencias a la geografia española o a los miembros de la familia real. Aludir a un nombre propio conocido parece ser uma necesidad que parte de una voluntad de acortar distancias e inventar puntos de referencia que permitan conjurar los temores provocados por los reveses e imprevisibilidades del viaje. Es también una forma eficaz de tornar verosímil y comprensible una geografia que los distantes lectores deben recrear a partir de palabras y equivalencias (recordemos cuando, para dar uno de tantos ejemplos, Colón describe los árboles "tan verdes y tan hermosos" comparándolos con el aspecto que en España presentan en el mes de mayo). Pero la evocación desempeña claramente otro papel, que va más allá de las funciones del relato o de las eventuales necesidades del narrador: formalizar, mediante la imposición del nombre conocido, la apropiación en favor de la corona de los territorios visitados. En este caso, esas tres funciones del nombre (conjurar, describir, apropiarse) parecen evidentes: en una isla 
denominada Española se funda una ciudad que se llama La Isabela, esa que los libros de historia reconocen como la primera villa española en las Américas.

La fundación ocurre en los últimos días de ese año 1493. Colón no evita la hipérbole al describir el lugar elegido para el emplazamiento. En la Carta Relación del segundo viaje, destaca la importancia del rio, con una "vega muy graciosa", y al que define como "mejor que el Guadalquivir". "Dende la villa al poniente, grandes dos leguas, es toda plaia mui fermosa, y al cavo un puerto de los mejores del mundo, en que cabrán todas las naos que en él ay". ${ }^{8}$ Otro de los tripulantes, en cambio, el doctor Diego Alvarez Chanca, dejó en su carta un testimonio menos exaltado. Chanca escribe: "Dicen que la carne del hombre es tan buena que no hay tal cosa en el mundo; y bien paresce porque los huesos que en estas casas hallamos todo lo que se puede roer todo lo tenían roido, que no había en ellos sino lo que su mucha dureza no se podía comer. Allí se halló en una casa cociendo en una olla un pescuezo de un hombre. Los mochachos que cativan córtanlos el miembro, é sírvense dellos fasta que son hombres, y después cuando quieren facer fiesta mátanlos é cómenselos, porque dicen que la carne de los mochachos é de las mojeres no es buena para comer". Después de haber dado profusas noticias sobre los Caribes y sus compulsivos hábitos antropofágicos ${ }^{9}$, y ante las sombrías informaciones de la destrucción de Navidad, el lugar de todas maneras le debe haber parecido una especie de refugio, al que define como "el mejor sitio y dispusición que pudiéramos escoger, donde hay mucho buen puerto é gran pesqueria". Pero también afirma que "Verdad sea que la tierra no consiente que se guarde de un día para otro porque es callente y húmeda, é por ende luego las cosas introfatibles ligeramente se corrompen. La tierra es muy gruesa para todas cosas: tiene junto un río prencipal é otro razonable, asaz cerca de muy singular agua". Lejos de alli, Pedro Mártir, guiado por las informaciones recibidas y por la imaginación multiplicada por la distancia, informa que "La naturaleza de aquella tierra es exhuberantemente
${ }^{8}$ Ver Varela, Consuelo, op.cit, p. 460.

${ }^{9}$ Ver Carta de Diego Alvarez Chanca, disponible en versión integral en http:// www.fortunecity.com/ victorian/churchmews/ 1216/Chanca.html. 
${ }^{10}$ Pedro Martir de Anglería, op. cit. Epistola 152, a Pomponio Leto, p. 44-46.

"Ver León Guerrero, M, op. cit., p. 254. feliz", y no vacila en incluir a Colón en el linaje de los fundadores ilustres:

Colón ha comenzado a edificar una ciudad (...) y a sembrar nuestras semillas y a criar animales de los nuestros. ¿Cómo hemos de admirar, ya que los Saturnos, las Ceres y los Triptolemos enseñaran nuevos inventos a los hombres, o que los fenicios edificaran a Sidón y a Tiro, o que los mismos tirios para habitar otras regiones emigrasen a tierras extrañas, fundasen nuevas ciudades y creasen nuevos pueblos? ${ }^{10}$

Como los personajes míticos de la epopeya y de la historia, Colón, apremiado por la urgencia de resguardar a la numerosa tripulación y por la necesidad de encontrar oro lo más rápidamente posible, comienza a dar formas a la nueva ciudad. En esto sigue fielmente las ya mencionadas "Instrucciones" del 29 de mayo de 1493 que había recibido de los Reyes Católicos, donde se le requería la construcción de los principales edificios públicos y el nombramiento de los oficiales (alcaldes, alguaciles, regidores) para ejercer el gobierno de la ciudad. Las Casas informa que

Puso suma diligencia em edificar luego casa para los bastimentos y municiones del armada, e iglesia y hospital, y para su morada una casa fuerte, según se pudo hacer; y repartió solares, ordenando sus calles y plaza, y avecínanse las personas principales y manda que cada uno haga su casa como mejor pudiere; las casas públicas se hicieron de piedra, las demás cada uno hacia de madera y paja. ${ }^{11}$

Las "Instrucciones" enfatizaban la importancia de construir una aduana, desde donde se pudiese llevar un riguroso control de todas las provisiones, que debían ser registradas en libro. La iglesia, donde sería celebrada la primera misa, el dia 6 de enero de 1494, responde al objetivo de evangelizar a los nativos y al pedido de las Instrucciones para que los "traten muy bien y amorosa- 
mente a los dichos indios sin que les hagan enojo alguno, procurando que tengan los unos con los otros mucha conversación y familiaridad". ${ }^{12}$ El hospital atendería a la numerosa tripulación agobiada por la pobre alimentación y las penurias. La casa fuerte serviría de residencia al própio Colón. Tales edificios estarían unidos por un trazado urbano que aparentemente contaría con una plaza como centro de referencia. El conjetural médico catalán Guillermo Coma, que hacía parte de la tripulación del segundo viaje, afirma en una carta que la ciudad "tiene uma ancha calle trazada a cordel [...] que es cortada después, transversalmente, por otras muchas costaneras". Con probable exageración añade que "en la plaza se alza un magnífico castillo con una elevada fortaleza”, y anuncia con incondicional optimismo que la ciudad "por aventajar a todas las demás em virtud de su estratégica situación y la benignidad de su clima, será dentro de muy pocos años populosa y repleta y frecuentada de colonos; competirá con cualquiera de las ciudades españolas cuando estén acabados los edificios y levantados sus magníficos muros". ${ }^{13}$ Ya para otro de los cronistas allí presentes, el italiano Miguel de Cuneo, la ciudad no pasaba de un sórdido casarío abandonado a su propia suerte. ${ }^{14}$

Con el tiempo, la convivencia entre indigenas y españoles se fue deteriorando en la villa y en el resto de la isla. Cada vez más lejos de las relaciones de "conversación y familiaridad" deseadas por los Reyes en sus Instrucciones, las tensiones se iban multiplicando. No hubo rápidas conversiones al cristianismo, como Colón había imaginado, y ni siquiera se había podido promover algún tipo de evangelización, ante las dificultades de comunicación y la falta de intérpretes. La escasez de alimentos era notoria y alarmante. Proliferaban las enfermedades, entre ellas las venéreas, debido al "tracto de la mugeres, que acá hallan abondoso; y si son deshonestos y deshordenados, no es maravilla que ayan pena", según condena el Almirante en su Carta relación. Del oro tan ansiado, apenas habían aparecido unas pocas muestras, que parecían más espejismos que certezas, y en nada se correspondían con las ilusiones
${ }^{12}$ Instrucciones del segundo viaje, en Morales Padrón, Francisco. Teoria y leyes de la conquista. Madrid: Cultura Hispánica, 1979, p. 67.

${ }^{13}$ Ver Coma, Guillermo. "Sobre las islas recientemente descubiertas del Mar Meridiano e Índico", en Cartas de particulares a Colón. Relaciones coetáneas. Editadas por Juan Gil y Consuelo Varela (Madrid, 1984). La cita fue extraída de la obra de Carlos Dobal Como pudo ser la Isabela., pp. 50-51

${ }^{14}$ Ver Varela, Consuelo. "La Isabela. Vida y ocaso de una ciudad efimera", en Revista de Indias, 1987, vol.

XLVII, núm 181, pp. 733-744, p. 737. 
${ }^{15}$ Ver Oliva de Coll, Josefina. La resistencia indigena ante la conquista. México: Siglo XXI Editores, 2003, p.19-32. de inmediata y fácil riqueza de los invasores. Expediciones habían sido enviadas hacia el sur, en busca de las minas del Cibao, seguidas por el establecimiento de una línea de fortalezas en el interior para asegurar el control militar y económico de la región. Para completar el cuadro de desventuras, surgen disensiones entre los europeos, como la rebelión encabezada por Bernal Díaz de Pisa ya en febrero de 1494, y, a continuación, en marzo de ese año, un incendio llega a destruir dos tercios de la precaria ciudad. $\mathrm{Al}$ año siguiente, Isabela sería duramente castigada por un huracán.

Para los indígenas, la presencia española en la isla solo había traido perjuicios. Las raices y peces que conformabam su alimentación comenzaban a escasear, ahora que había muchas otras bocas interesadas en tales recursos.Víctima de las enfermedades, de los tributos y de los "rescates" que comenzaban a practicarse sistemáticamente, la población nativa padecía las irreversibles consecuencias de un choque cultural que no estaba preparada para soportar. Y aunque se ejercen varios tipos de resistencia, desde la negativa de cultivar las tierras hasta la confrontación, y se forma una coalición de caciques para organizar el rechazo al avance español, las fuerzas indígenas son vencidas en sucesivas expediciones. ${ }^{15}$ Los ejemplos de violencias sufridas, que inauguran la larga lista de masacres que tantas veces se repetirán durante el siglo XVI en todo el continente americano, son numerosos y conocidos. Aparecen en los propios textos colombinos, en la Relación de Miguel de Cuneo de 1495 (donde el propio autor describe cómo violó a una nativa), y alcanzan su paroxismo en la extensa enumeración de atrocidades que Las Casas menciona en los dos capítulos relativos a la isla Española de su Brevísima relación.

El malestar existente en la ciudad aumentaría durante la ausencia de Colón, que entre abril y septiembre de ese 1494 estuvo explorando las costas de Cuba y Jamaica. Sin oro, y con hambre, la situación de los habitantes se hacía insostenible. En su Historia General y Natural de las Indias, Oviedo recuerda que gran parte de los españoles 
murió, y otros tuvieron que sobrevivir comiendo los pocos perros que restaban, como también iguanas, lagartijas y culebras. ${ }^{16}$ El cuadro tendió a empeorar en los años siguientes, durante el gobierno de Bartolomé Colón, el hermano del Almirante. Cuando Colón retorna a España, en marzo de 1496, los Reyes ya cuentan con los informes negativos del padre Boyl y de Margarit, que critican la administración colombina y cuestionan la supuesta abundancia de oro que había sido lema permanente del Almirante. En el período que se extiende desde su regreso a España hasta la nueva llegada a América en su tercer viaje, en agosto de 1498, la decadencia de Isabela se torna irreversible. Para ese entonces, el puerto de la Isabela había sido abandonado definitivamente, y sus funciones como principal núcleo urbano y administrativo se habían trasladado al sur de la isla, en la que vendría a ser la ciudad de Santo Domingo.

Así, menos de cinco escasos años separaron el comienzo y el fin de La Isabela. Del lugar quedaron algunos restos arqueológicos, que continúan siendo analizados hasta hoy. ${ }^{17}$ Quedaron también leyendas, como la que cuenta Las Casas, sobre algunos fantasmas vestidos con trajes españoles que solían aparecer por entre las ruinas de la ciudad, y que, cuando eran interrogados, permanecían en silencio y se limitaban a saludar, "[quitando] juntamente con los sombreros las cabezas de sus cuerpos, quedando descabezados y luego desa-parecieron". ${ }^{18}$ Esa ciudad apenas poblada por fantasmas, ciudad maldita, cuya memoria debia ser borrada cuanto antes, representa la distopía del sueño colombino: la ciudad que no fue, que se esfumó casi antes de haber sido, la ciudad que permanecerá para siempre ausente. Apenas años después de haber sido fundada, ya les parecía a sus contemporáneos algo remoto y ligeramente ilusorio. En los mapas de las primeras décadas del siglo XVI, La Isabela aparece localizada en sitios tan dispares como la isla de Cuba o La Florida, y se la confunde frecuentemente con la pequeña isla del mismo nombre (llamada Crooked, hoy parte de las Bahamas) que Colón bautizó en su primer viaje. Pero La Isabela, ese
${ }^{16}$ Fernandez de Oviedo, Gonzalo. Historia Natural y General de las Indias.Madrid, BAE, 1992. Libro II, cap. XIII, p. 48.

${ }^{17}$ Las investigaciones han sido llevadas a cabo en estos ultimos años por el Florida Museum of Natural History (ver http:// www.flmnh.ufl.edu/ histarch/Isabela.htm), que complementan cuidadosas expediciones cumplidas durante el siglo XX (ver la obra de Carlos Dobal ya citada para otras informaciones).

${ }^{18}$ La leyenda aparece en la Historia general de las Indias, de Las Casas, y la recoge Consuelo Varela em "La Isabela. Vida y ocaso de una ciudad efimera", op. cit, p 741. 
nombre que recuerda que "no existe tal lugar", en realidad continuaría apareciendo muchas otras veces a lo largo del período colonial, como desatino, esperanza y fracaso. La Isabela, que fue real y dejó rapidamente de serlo, sería tan ilusoria como aquellas otras ciudades que se multiplicarían para desvanecerse por la geografía americana, como la ciudad de los Césares, las siete de Cibola, Paititi, El Dorado. Ciudades de sueño, plenamente imaginarias, que nunca fueron, pero que permanecen y duran apenas como algo fugitivo, deseado y en definitiva invisible: una ficción, una quimera. 


\section{Referências}

ALVAREZ CHANCA, Diego. "Carta", disponible en versión integral en http://www.fortunecity.com/victorian/ churchmews/1216/Chanca.html.

COMA, Guillermo. "Sobre las islas recientemente descubiertas del Mar Meridiano e Índico", en Cartas de particulares a Colón. Relaciones coetáneas. Editadas por Juan Gil y Consuelo Varela. Madrid, 1984.

DOBAL, Carlos Como pudo ser la Isabela. Santiago, República Dominicana: Pontificia Universidad Católica Madre y Maestra, 1998.

FERNANDEZ DE OVIEDO, Gonzalo. Historia Natural y General de las Indias. Madrid, BAE, 1992.

IRVING, Washington. Vida y viajes de Cristóbal Colón. Madrid: Gaspar y Roig Editores, 1851.

LAS CASAS, Bartolomé. Obras completas. v. 14. Madrid: Alianza, 1989.

LEÓN GUERRERO, Maria Monserrat. El segundo viaje colombino. Alicante: Biblioteca Virtual Miguel de Cervantes, 2002.

MARTIR DE ANGLERIA, Pietro Cartas sobre el Nuevo Mundo. Madrid: Polifemo, 1990.

MORALES PADRÓN, Francisco. Teoria y leyes de la conquista. Madrid: Cultura Hispánica, 1979.

OLIVA DE COLL,Josefina. La resistencia indígena ante la conquista. México: Siglo XXI Editores, 2003.

SANS CAMAÑES, Porfírio. Las ciudades en la América Hispana. Siglos XV al XVIII. Madrid: Silex, 2004.

VARELA,Consuelo. Cristóbal Colón. Textos y documentos completos. Madrid: Alianza, 1997

. "La Isabela. Vida y ocaso de una ciudad efímera". In:

Revista de Indias, 1987, v.. XLVII, núm 181, p. 733-744. 